\title{
On a Method for Solving the Inverse Problem in Potential Scattering
}

\author{
M. BLAŽEK \\ ČSAV Fyzikálny ústav SAV, Bratislava, Czechoslovakia
}

\begin{abstract}
A method for solving the inverse problem in the non-relativistic elastic scattering theory, using the analytic and asymptotic properties of the scattering amplitude is proposed and the influence of the discontinuity parameters of the scattering amplitude on the properties of the resulting potentials is discussed. The case with spherically symmetric forces and without bound states is considered. The possibility for solving the inverse problem by this method, leading to the singular repulsive potentials is mentioned.
\end{abstract}

\section{Introduction}

The present investigations in the non-relativistic potential scattering can be divided into two groups, i) the direct investigations where a definite class of potentials is assumed and the properties of some investigated scattering functions are to be determined and, ii) the inverse investigations proceeding from the known scattering quantities and the acting forces in the Schrödinger (or Schrödinger-like) equation are to be found.

In the first mentioned group of investigations, usually the characteristic properties of one of the following functions are determined: the scattering amplitude $A(k, \cos \vartheta)$ ( $k$ is the c.m. momentum and $\vartheta$ the c.m. scattering angle), the partial wave scattering amplitude $A_{l}(k)$ ( $l$ is the physical angular momentum), the Regge function $A(\lambda, k)(\lambda$ is the complex angular momentum), the Jost function $f_{l}(k)$ and/or $f(\lambda, k)$, the conical amplitude, etc., in general an arbitrary scattering function or its coefficients in a series expansion. In this direction, there were investigated e.g. the analytic properties of the various scattering amplitudes in various complex domains, the validity of the Mandelstam representation for the scattering amplitude or of an integral representation for the Jost function or for the conical amplitude or for the Regge parameters, customarily, considering the class of Yukawa forces [1]. Some survey papers deal with these problems (see e.g. [2]).

In the second of the above mentioned group of investigations one proceeds from the known properties of the scattering amplitude or of any function whose properties are derivable from the latter, and the 
methods of finding the scattering potentials in the Schrödinger equation are to be established. The investigations in this direction, taking into account also the application of some approximation methods are in details less elaborated than in the former case. First investigations were originated by C. Møller, C. E. Fröberg, N. Levinson, E. HylleraAs and others. More details can be found in the survey paper [3]; see also [4].

The solution of the inverse problem is interesting also from an other point of view, namely if the scattering process is described by the scattering amplitude which can be determined by more independent ways, the problem of mutual internal connections of these ways there arises. The solution of the inverse problem contributes to the understanding of this connection.

The potential can be obtained from the scattering quantities, schematically by means of the following three methods,

a) the first Born approximation, or any other series expansion which relates the potential with the scattering quantity (we include here also the method of J. Charap and S. FubinI, and of others),

b) the direct use of the Schrödinger equation with some Ansatz (as it is customary for the Jost solution); these works apply usually the original methods of V. BargmanN [5],

c) the analytic properties of the scattering functions under consideration in certain complex domains.

For instance, using the analytic properties of the Jost functions $f_{l}(k)$ or $f(\lambda, k)$ in the $k$ - or $\lambda$-complex plane, the solution of the inverse problem follows usually the original papers of I. M. GELFAND and B. M. Levitan, and R. Jost and W. KoHn [6] (see also [7]) where the spectral function technique is applied for the problem under consideration. This is demonstrated e.g. also in [8], where the inverse problem in the complex angular momentum plane is solved. However, an other method for determination of the potential is established in [9]; the analytic properties of the zero angular momentum partial wave scattering amplitude in connection with the Fourier transform technique are there used. The generalization for higher waves by means of the Hankel transform can be found in [10]. By means of an Ansatz for the Regge function the problem is partially solved in [11] where the first Born approximation is used for the determination of the potential.

The different ways of the solution of the inverse problem lead to the different classes of potentials. The peratization technique used in the last years, pointed out the convenience for finding the solution of the inverse problem for potentials singular and repulsive at small distances; the recent direct investigations in this field are rather extensive. Since only the Gelfand-Levitan's procedure is usually used for solving the inverse problem in what follows we emphasize the other procedure (be$20^{*}$ 
longing to the methods mentioned above sub c)) allowing to determine the potential directly by means of the basic properties of the scattering amplitude. It would be convenient to extend this method also for problems leading to the singular potentials.

After the next section of preliminary relations we derive the basic integral equation in the Sec. III. The discussion is performed in the last section.

\section{Preliminary relations}

1. The Schrödinger equation $\hat{H} \psi=\hat{T} \psi+\hat{U} \psi=E \psi$ leads for the spherically symmetric potentials $V=V(r)$ to the equation $(\hbar=2 m=1)$

where

$$
\frac{d^{2} u_{l}(k, r)}{d r^{2}}+\left[k^{2}-\frac{l(l+1)}{r^{2}}-V(r)\right] u_{\imath}(k, r)=0
$$

$$
\psi=\sum_{l=0}^{\infty}(2 l+1) \frac{u_{l}(k, r)}{r} P_{l}\left(\mathbf{n}_{1} \cdot \mathbf{n}_{2}\right)
$$

(the $P_{l}$ 's are the Legendre polynomials, $\mathbf{n}_{1}$ is the direction of the ingoing and $\mathbf{n}_{\mathbf{2}}$ of the outgoing particle). It is obvious that the non-local interaction of the form

$$
\hat{U} \psi=\int_{\Omega_{\mathbf{n}}} \mathscr{U}\left(r ; \mathbf{n}_{1} \cdot \mathbf{n}\right) \psi\left(k, r ; \mathbf{n} \cdot \mathbf{n}_{2}\right) d \Omega_{\mathbf{n}}
$$

leads also to the same radial equation (1), the only difference being now in the $l$-dependence of the potential $V_{l}(r)$. This is seen using in (3) the series expansion (2) and ${ }^{1}$

$$
\mathscr{U}\left(r ; \mathbf{n} \cdot \mathbf{n}_{2}\right)=\sum_{l=0}^{\infty}(2 l+1) V_{l}(r) P_{l}\left(\mathbf{n}_{1} \cdot \mathbf{n}_{2}\right) .
$$

Therefore, solving the inverse problem for each partial wave separately we obtain a potential for each partial wave and this leads to the nonlocal interaction of the form (3). In general, one does not know the conditions securing the locality of the interaction, obtained by solving the inverse problem for each partial wave. Usually it is assumed only that the non-locality is weak.

2. It is customary to consider the potentials with finite first and second absolute moments [2]. In this case we can express the solution of the radial equation ( 1$)$ by means of the free particle $(V \equiv 0)$ solution $u_{l}^{(0)}(k, r)$

$$
u_{l}(k, r)=u_{l}^{(0)}(k, r)+\int_{r}^{a} K_{l}(r, t) u_{l}^{(0)}(k, t) d t .
$$

1 From the latter, non-local case expressed by eq. (4), the former, local case follows using the relations $V_{l}(r) \rightarrow V(r)$ and $\sum_{l=0}^{\infty}(2 l+1) P_{l}\left(\mathbf{n}_{1} \cdot \mathbf{n}_{2}\right)=4 \pi \delta\left(\mathbf{n}_{1}-\mathbf{n}_{2}\right)$. 
In (5) $a$ is a fixed boundary value and $K_{l}(r, t)$ is the kernel of this integral transform. Introducing (5) into (1) we get

$$
\begin{gathered}
\frac{\partial^{2} K_{l}(r, t)}{\partial r^{2}}-\frac{l(l+1)}{r^{2}} K_{l}(r, t)-\left[\frac{\partial^{2} K_{l}(r, t)}{\partial t^{2}}-\frac{l(l+1)}{t^{2}} K_{l}(r, t)\right] \\
=V(r) K_{l}(r, t), \\
V(r)=-2 \frac{d K_{l}(r, r)}{d r}
\end{gathered}
$$

(in general, $V=V_{l}(r)$ ) and the boundary condition

$$
\lim _{t \rightarrow a}\left[u_{l}^{(0)}(k, t) \frac{\partial K_{l}(r, t)}{k \partial t}-K_{l}(r, t) \frac{\partial u_{l}^{(0)}(k, t)}{k \partial t}\right]=0 .
$$

In the Gelfand-Levitan's procedure, the transformation (5) by means of the regular solution is used. In this case $u_{l}^{(0)}(k, r)=k r j_{l}(k r) \sim$ $\sim \varphi_{l}^{(0)}(k, r)^{2}$, and further $a=0, K_{l} \rightarrow-K_{l}$. The $j_{l}$ 's are the spherical Bessel functions.

If the Jost solutions $f_{l}(k, r)$ of the radial equation (1) are to be used, the more natural way in this case is to put in (5) $a=\infty$ and $u_{l}^{(0)}(k, r)$ $=i^{-(l+1)} k r h_{l}^{(2)}(k, r) \equiv f_{l}^{(0)}(k, r)^{3}$. The spherical Hankel functions of the second kind $h_{l}^{(2)}(z)$ are given by

$$
z h_{l}^{(2)}(z)=i^{l+1} e^{-i z} \sum_{s=0}^{l} \frac{1}{2^{s}(s !)} \frac{(l+s) !}{(l-s) !} \frac{1}{(i z)^{s}} .
$$

The transformation (5) has now the form

$$
f_{l}(k, r)=f_{l}^{(0)}(k, r)+\int_{r}^{\infty} K_{l}(r, t) f_{l}^{(0)}(k, t) d t .
$$

By means of (8) the influence of the repulsive barrier term in the radial equation is separated.

3. Let us consider an integral equation of the type

$$
K_{l}(r, \sigma)+\int_{r}^{a} K_{l}(r, t) F_{l}(t, \sigma) d t+F_{l}(r, \sigma)=0
$$

where $K_{l}(r, \sigma)$ is an unknown function and $F_{l}(r, \sigma)$ is given by

$$
F_{l}(r, \sigma)=\mathrm{const} \int_{A}^{B} M_{l}(k) u_{l}^{(0)}(k, r) u_{l}^{(0)}(k, \sigma) d k .
$$

In (10) $A$ and $B$ are fixed and $M_{l}(k)$ is an arbitrary (in practice a known) function. Substituting (10) into (9) we can show by direct computation

${ }^{2}$ We use the regular solution $\varphi_{l}(k, r)$ of $(1)$ defined by $\lim _{r \rightarrow 0}\left[r^{-(l+1)} \varphi_{l}(k, r)\right]=1$.

${ }^{3}$ We use the Jost solution $f_{l}(k, r)$ of $(1)$ normalized by $\lim _{r \rightarrow \infty}\left[f_{l}(k, r) \exp (i k r)\right]=1$; $f_{l}^{(0)}(k, r)$ is the free-particle Jost solution. 
that the following equation holds

$$
\begin{aligned}
\frac{\partial^{2} K_{l}(r, \sigma)}{\partial r^{2}}-\frac{l(l+1)}{r^{2}} K_{l}(r, \sigma)-\left[\frac{\partial^{2} K_{1}(r, \sigma)}{\partial \sigma^{2}}\right. & \left.-\frac{l(l+1)}{\sigma^{2}} K_{l}(r, \sigma)\right]= \\
& =-2 \frac{d K_{l}(r, r)}{d r} K_{l}(r, \sigma)
\end{aligned}
$$

with the boundary condition

$$
\lim _{t \rightarrow a}\left[u_{l}^{(0)}(k, t) \frac{\partial K_{l}(r, t)}{k \partial t}-K_{l}(r, t) \frac{\partial u_{l}^{(0)}(k, t)}{k \partial t}\right]=0 .
$$

Hence, if we define

$$
-2 \frac{d K_{l}(r, r)}{d r}=V(r)
$$

then the relations (6) are reproduced. It is obvious that from (11) we get the Schrödinger radial equation (1) with the appropriate boundary condition.

The solution of the inverse problem by means of the methods mentioned in the introduction in category c), leads usually to the integral equation of the form (9). However, introducing now a priori the equation (9) with the definition of the potential (11 c), we obtain the Schrödinger equation (1) and its solution in the form (5).

4. We introduce the functions $\mathscr{K}, \mathscr{F}$ and $\mathscr{N}$

$$
\begin{aligned}
\mathscr{K}\left(r, \sigma ; \mathbf{n}_{1} \cdot \mathbf{n}_{2}\right) & =\Sigma(2 l+1) K_{l}(r, \sigma) P_{l}\left(\mathbf{n}_{1} \cdot \mathbf{n}_{2}\right) \\
\mathscr{F}\left(r, \sigma ; \mathbf{n}_{1} \cdot \mathbf{n}_{2}\right) & =\Sigma(2 l+1) F_{l}(r, \sigma) P_{l}\left(\mathbf{n}_{1} \cdot \mathbf{n}_{2}\right) \\
\mathscr{N}\left(r, \sigma ; k ; \mathbf{n}_{1} \cdot \mathbf{n}_{2}\right) & =\Sigma(2 l+1) u_{l}^{(0)}(k, r) u_{l}^{(0)}(k, \sigma) P_{l}\left(\mathbf{n}_{1} \cdot \mathbf{n}_{2}\right)
\end{aligned}
$$

(we sum up from $l=0$ to $l=\infty$ ). The basic integral equation (9) can be expressed by means of (12) in the form

$$
\begin{gathered}
\mathscr{K}\left(r, \sigma ; \mathbf{n}_{1} \cdot \mathbf{n}_{\mathbf{2}}\right)+\int_{r}^{a} d t \int_{\Omega_{\mathbf{n}}} \frac{d \Omega_{\mathbf{n}}}{4 \pi} \mathscr{K}\left(r, t ; \mathbf{n}_{1} \cdot \mathbf{n}\right) \mathscr{F}\left(t, \sigma ; \mathbf{n} \cdot \mathbf{n}_{\mathbf{2}}\right)+ \\
+\mathscr{F}\left(r, \sigma ; \mathbf{n}_{\mathbf{1}} \cdot \mathbf{n}_{\mathbf{2}}\right)=0
\end{gathered}
$$

where

$$
\mathscr{F}\left(r, \sigma ; \mathbf{n}_{1} \cdot \mathbf{n}_{2}\right)=\operatorname{const} \int_{A}^{B} d k \int_{\Omega_{\mathbf{n}}} \frac{d \Omega \mathbf{n}}{4 \pi} \mathscr{M}\left(k, \mathbf{n}_{1} \cdot \mathbf{n}\right) \mathscr{N}\left(r, \sigma ;-k ; \mathbf{n} \cdot \mathbf{n}_{2}\right)
$$

and

$$
\mathscr{M}\left(k, \mathbf{n}_{\mathbf{1}} \cdot \mathbf{n}_{\mathbf{2}}\right)=\sum_{l=0}^{\infty}(2 l+1) M_{l}(k) P_{l}\left(\mathbf{n}_{\mathbf{1}} \cdot \mathbf{n}_{\mathbf{2}}\right) .
$$

The potential function (4) is now given by

$$
\mathscr{U}\left(r ; \mathbf{n}_{\mathbf{1}} \cdot \mathbf{n}_{2}\right)=-2 d \mathscr{K}\left(r, r ; \mathbf{n}_{1} \cdot \mathbf{n}_{2}\right) / d r .
$$

In the Gelfand-Levitan's case (see e.g. [6], [8]) there is in (10)

$$
M_{l}(k)=\left[\frac{1}{\left|f_{l}(k)\right|^{2}}-\frac{1}{\left|f_{l}()^{\prime}(k)\right|^{2}}\right] k^{-2 l} ; A=0, B=\infty .
$$


The $f_{l}(k)$ 's are the Jost functions and for the free particle case we have $f_{l}^{(0)}(k)=[(2 l+1) ! !] /(i k)^{l}$. We see that the quantity $(15)$ has in this case no physical meaning. However, the procedure developed in the next section leads to the basic integral equation of the form (9) with the function $M_{l}(k)$ equal to the partial wave scattering amplitude $A_{l}(k)$. Hence, the function (15) is the scattering amplitude $\mathscr{A}(k, \cos \vartheta)$ and the inverse problem is solved by means of its basic properties.

\section{Derivation of the integral equation}

1. We proceed from the well known relation between the regular $\varphi_{l}(k, r)$ and the Jost $f_{l}(k, r)$ solutions of the radial equation (1)

$$
\varphi_{l}(k, r)=\frac{1}{2 i k}\left[f_{l}(k) f_{l}(-k, r)-f_{l}(-k) f_{l}(k, r)\right] .
$$

Introducing the $S$-matrix element by $S_{l}(-k)=(-1)^{l} f_{l}(-k) / f_{l}(k)$ we obtain from (16)

$$
\begin{aligned}
\frac{2 i k \varphi_{l}(k, r)}{f_{l}(k)} & =(-1)^{l+1} f_{l}(k, r)+f_{l}(-k, r)+(-1)^{l} f_{l}(k, r) \times \\
& \times\left[1-S_{l}(-k)\right] .
\end{aligned}
$$

We express the Jost solution $f_{l}(k, r)$ in the form (8)

$$
f_{l}(k, r)=i^{-(l+1)}\left[k r h_{l}^{(2)}(k r)+\int_{r}^{\infty} K_{l}(r, t) k t h_{l}^{(2)}(k t) d t\right]
$$

and from (17) we have

where

$$
\frac{2 k \varphi_{l}(k, r)}{i^{l} f_{l}(k)}-2 k r j_{l}(k r)=I_{1}+I_{2}+I_{3}+I_{4}
$$

$$
\begin{aligned}
& I_{1}=\int_{r}^{\infty} d t K_{l}(r, t) k t h_{l}^{(2)}(k t), \\
& I_{2}=(-1)^{l+1} \int_{r}^{\infty} d t K_{l}(r, t)\left[-k t h_{l}^{(2)}(-k t)\right], \\
& I_{3}=-k r h_{l}^{(2)}(k r)\left[1-S_{l}(-k)\right], \\
& I_{4}=-\int_{r}^{\infty} d t K_{l}(r, t) k t h_{l}^{(2)}(k t)\left[1-S_{l}(-k)\right] .
\end{aligned}
$$

After a short manipulation the resulting expression from (18) is

$$
\begin{aligned}
2 \int_{-\infty}^{+\infty} d k k^{l+1}\left[\frac{\varphi_{l}(k, r)}{(2 l+1) ! !}-\frac{k r j_{l}(k r)}{k^{l+1}}\right] k \sigma h_{l}^{(2)}(k \sigma)+ \\
+2 \int_{-\infty}^{+\infty} d k \frac{k^{l+1} \varphi_{l}(k, r)}{(2 l+1) ! !}\left[\frac{(2 l+1) ! !}{(i k)^{l} f_{l}(k)}-1\right] k \sigma h_{l}^{(2)}(k \sigma) \\
=\int_{-\infty}^{+\infty} d k\left[I_{1}+I_{2}+I_{3}+I_{4}\right] k \sigma h_{l}^{(2)}(k \sigma) .
\end{aligned}
$$


2. a) The left hand side of (19) is zero provided there are no bound states.

This can be demonstrated by closing the integral path on the l.h.s. of (19) in the lower momentum half plane by a large semicircle and using the relations ${ }^{4}$

$$
\begin{aligned}
& \left|k^{l+1} k \sigma h_{l}^{(2)}(k \sigma)\left[\frac{\varphi_{l}(k, r)}{(2 l+1) ! !}-\frac{k r j_{l}(k r)}{k^{l+1}}\right]\right| \rightarrow 0 \\
& \left|k \sigma h_{l}^{(2)}(k \sigma) k r j_{l}(k r)\left[\frac{f_{l}^{(0)}(k)}{f_{l}(k)}-1\right]\right| \rightarrow 0
\end{aligned}
$$

which are true for $|k| \rightarrow \infty, \operatorname{Im} k \leqq 0, r \leqq \sigma$.

In the enclosed region, the first integrand in the l.h.s. is a holomorphic function of the momentum $k$. From the second integrand only the zero points of the Jost function $f_{l}(k)$ in the lower momentum half plane can contribute. However, we assume there are no bound states present and therefore the Jost function $f_{l}(k)$ has no roots in this lower half plane. We have therefore the result that the second integrand in the l.h.s. is also a holomorphic function in this region.

b) The right hand side of (19) can be expressed as

$$
\begin{array}{r}
\int_{-\infty}^{+\infty} d k\left(I_{1}+I_{2}\right) k \sigma h_{l}^{(2)}(k \sigma)=2 \int_{0}^{\infty} d t K_{l}(r, t) \int_{-\infty}^{+\infty} d k k t j_{l}(k t) k \sigma j_{l}(k \sigma) \\
=2 \pi K_{l}(r, \sigma)
\end{array}
$$

and

$$
\int_{-\infty}^{+\infty} d k\left(I_{3}+I_{4}\right) k \sigma h_{l}^{(2)}(k \sigma)=2 \pi F_{l}(r, \sigma)+2 \pi \int_{r}^{\infty} d t K_{l}(r, t) F_{l}(t, \sigma)
$$

where

$$
F_{l}(r, \sigma)=-\frac{1}{2 \pi} \int_{-\infty}^{+\infty} d k\left[1-S_{l}(k)\right]\left[-k r h_{l}^{(2)}(-k r)\right]\left[-k \sigma h_{l}^{(2)}(-k \sigma)\right]
$$

c) Taking into account the results obtained for the 1.h.s. and for the r.h.s. of (19) we get the basic integral equation of the form (9)

$$
K_{l}(r, \sigma)+\int_{r}^{\infty} K_{l}(r, t) F_{l}(t, \sigma) d t+F_{l}(r, \sigma)=0, \quad r \leqq \sigma
$$

where the kernel $F_{l}(r, \sigma)$ is now given by $(21)$. As we consider the elastic scattering with the threshold condition $A_{l}(k) \sim k^{2 l}$ (for $k \rightarrow 0$ ), the integrand in (21) behaves well for small momenta.

Applying the procedure from the end of the preceding section we get the integral equation (13) with the kernel (14) in the form

$$
\mathscr{F}\left(r, \sigma ; \mathbf{n}_{\mathbf{1}} \cdot \mathbf{n}_{\mathbf{2}}\right)=-\frac{1}{2 \pi i} \int_{-\infty}^{+\infty} k d k \int_{\Omega_{\mathbf{n}}} \frac{d \Omega_{\mathbf{n}}}{4 \pi} \mathscr{A}\left(k, \mathbf{n}_{1} \cdot \mathbf{n}\right) \mathscr{N}\left(r, \sigma ;-k ; \mathbf{n} \cdot \mathbf{n}_{2}\right) \text {. }
$$

4 The relations (20) can be obtained by means of the relations $(3,9),(3,13)$ and $(4,16)$ of R. G. NEWTON's paper quoted above in [2]. 
In (23) the function $\mathscr{N}$ is known (see (12) with $u_{l}^{(0)}(k, r) \rightarrow k r h_{l}^{(2)}(k r)$ ). The scattering amplitude is given as

$$
\mathscr{A}(k, \cos \vartheta)=\sum_{l=0}^{\infty}(2 l+1)\left[\left(S_{l}(k)-1\right) / 2 i k\right] P_{l}\left(\cos \vartheta=\mathbf{n}_{\mathbf{1}} \cdot \mathbf{n}_{\mathbf{2}}\right)
$$

Enclosing the integral path in (23) by the large semicircle in the upper momentum half plane ${ }^{5}$, we see the only input information comes from the analytic properties of the scattering amplitude $\mathscr{A}(k, \cos \vartheta)$ in this half plane (this corresponds to the physical sheet of the Riemann energy surface, $E=k^{2}$ ) for physical values of the transferred momentum (i.e. $-1 \leqq \operatorname{Re} \cos \vartheta \leqq+1$ and $\operatorname{Im} \cos \vartheta=0$ ).

The potentials obtained by this method, in general, decrease exponentially at large distances. However, for particular values of the parameters specifying the singularities of the scattering amplitude, the potentials show a pathological behavior. The assertion that the potentials possess first and second absolute moments expresses that the above mentioned parameters are from certain domains.

\section{Discussion}

By means of the methods which solve the inverse problem we can find as customary uniquely the potential belonging to the scattering quantity under consideration. However, if the potential is required to have certain properties, we have to add some further conditions to the scattering quantity.

Suppose that for a given angular momentum the singularities of the partial wave scattering amplitude $A_{l}(k)$ lie on the positive imaginary axis in the momentum plane; then the nearest singularity determines the forces with the largest range, as follows from (21). The equations derived in the $n$-pole approximation for these upper singularities of the $s$-scattering amplitude are the same as obtained by the $N / D$ method from the dispersion relations [12]. We get in this $s$-scattering case the Hulthén's potential, the Noyes-Wong equation etc.; more details can be found in [13].

Considering further this $n$-pole approximation, the influence of the values of the pole parameters in the redundant poles (always lying in the upper momentum half plane) on the occurence of the bound state poles can be investigated. This problem is partially solved in [14] where the domains of the redundant poles parameters inducing no bound state poles are established. A more general discussion is performed in [13]. On the other hand, let us consider a branch cut on the positive imaginary

${ }^{5}$ We assume that the exponential damping factors arising from the spherical Hankel functions (7) in $\mathscr{N}$ suffice to insure the convergence of the integral (23) on the mentioned large semicircle. 
axis. The discontinuity across this cut, specifying the potential (the "redundant discontinuity") can cause an allowed energy band, in general, a band spectrum can arise. The inverse problem for this case is still unsolved.

The influence of the parameters specifying the redundant singularities can be illustrated very simply in the one-pole approximation. Let us consider, for instance, the low-energy neutron-proton elastic scattering in the relative $s$-state described in the effective range approximation by the relation: $k \cot \delta=-v+\left(r_{0} k^{2}\right) / 2,0<r_{0}\left(\delta\right.$ is the $s$-phase shift, $v^{-1}$ determines the scattering length and $r_{0}$ is the effective range). In this case the $S$-matrix element is $S_{0}(k)=f(k) / f(-k)$ with the Jost function $f(k)=(k+i b) /(k-i a)$ where $a=\left[1+\left(1-2 v r_{0}\right)^{1 / 2}\right] / r_{0}$ and $b=-[-1+$ $\left.+\left(1-2 v r_{0}\right)^{1 / 2}\right] / r_{0}$. As we see $a$ is always positive. For $k=i a$ we have a redundant pole in the $S$-matrix. Introducing the "coupling constant" $\tau$ by means of the residuum in the redundant pole $\tau=(2 i a)^{-1} \operatorname{Res}_{k=i a} S_{0}(k)$ $=(a+b) /(a-b)$ we obtain $b=a(\tau-1) /(\tau+1)$. Regarding the last relation $b=b(a, \tau)$ we get $S=S(k ; a, \tau)$. We see that the sign of $b$ depends on the value of $\tau$, namely whether $|\tau| \gtrless 1$ (both possibilities are consistent with unitarity). If $\tau>1$, we have in the upper half $k$-plane a further pole in the $S$-matrix and this pole corresponds to a true bound state (triplet scattering). The condition for atractivness of the corresponding potential has the form $0<\tau$. Hence, for $0<\tau<1$ the attractive potential has no bound state (singlet scattering ${ }^{6}$ ) since the corresponding pole lies in the lower momentum half plane (and nearer to the origin than the redundant pole). Therefore, if we know that the function $S_{0}(k)$ has in the upper half plane one pole $k=i a$ with the corresponding residuum or coupling constant $\tau$, we need a further information in order to be sure that this pole is the only one present in the upper half plane ${ }^{7}$.

The generalization of the procedure of Sec. III. to the relativistic case (considering e.g. the radial part of the Klein-Gordon or of the Dirac

${ }^{6}$ For the singlet scattering we have from experiment $v^{-1} \doteq-23.7 \cdot 10^{-13} \mathrm{~cm}$ and $r_{0} \doteq 2.40 \cdot 10^{-13} \mathrm{~cm}$. We get $a=0.87 \cdot 10^{13} \mathrm{~cm}^{-1}$ and $b=-0.04 \cdot 10^{13} \mathrm{~cm}^{-1}$. From the definition of $\tau=(a+b) /(a-b)$ we see the relation $0<\tau<1$ is true. In this case, by means of (22) we obtain the known Eckart's potential $V(r)$ $=-8 a^{2} \tau[\exp (-2 a r)] /[1+\tau \exp (-2 a r)]^{2}$.

7 We note that for the ratio of the residue in the redundant pole $k=i a(a>0)$ and in the pole $k=i b$ we obtain: $\left[\operatorname{Res}_{k=i a} S_{0}(k)\right] /\left[\operatorname{Res}_{k=i b} S_{0}(k)\right]=-a / b$. If the pole $k=i b$ corresponds to a true bound state, i.e. $b>0$, the two mentioned residues have opposite signs. Characterizing a pole by a strength which is proportional to the residue in this pole, we have the result that in this case the strength in the bound state pole has the opposite sign to that of the redundant pole. This is in agreement with [15]. 
equations) can be also performed. However, in this case the resulting potentials are energy dependent. This leads to the discussion of the subject of "potential scattering" (as it was introduced in [16]) and also to the considerations on the basic properties which the potential itself would have (this is performed in more detail e.g. in [17]).

Acknowledgement. The author is grateful to Professor J. Hamilton for reading the manuskript and to him and the NORDITA, Copenhagen, Danmark, for their kind hospitality.

\section{References}

[1] Blankenbecler, R., M. L. Goldberger, N. N. Khuri, and S. B. Treiman: Ann. Phys. 10, 62 (1960). - Fivel, D.: Phys. Rev. 125, 1085 (1962). de Alfaro, V., T. Regge, and C. Rossettis: Nuovo cimento 26, 1029 (1962). - Petrášs, M.: Nuovo cimento 31, 247 (1964).

[2] Newton, R. G.: J. Math. Phys. 1, 319 (1960). - Martin, A.: Nuovo cimento Suppl. 21, 157 (1961), $3^{\circ}$ Trim; and also: Analyticity in potential scattering [published in the Progress in Elementary Particle and Cosmic Ray Physics 8, 1 (1965)]. - REgGe, T.: In Theoretical physics, dir. by A. SALAM (lectures presented at a seminar, Trieste 1962) IAEA Vienna 1963. - BeLINFANTE, J. G., and B. C. UnaL: J. Math. Phys. 4, 372 (1963).

[3] Faddeyev, L. D.: J. Math. Phys. 4, 72 (1963) (translated from UMN SSSR 14, No. 4(88), 57 (1959)).

[4] Swan, P., and W. A. Pearce: Deduction of potentials from scattering phase shifts (to be published in Nuclear Phys.).

[5] Bargmann, V.: Phys. Rev. 75, 301 (1949); Rev. Mod. Phys. 21, 488 (1949).

[6] Gelfand, I. M., and B. M. Levitan: IAN SSSR (ser. mat.) 15, 309 (1951) [English translation in Amer. Math. Soc. Trans., Sec. 21, 250 (1955)]. Jost, R. and W. Kohn: Dan. Mat. Fys. Medd. 27, No. 9 (1953). - Levinson, N.: Phys. Rev. 89, 755 (1953).

[7] Hylleraas, E. A.: Ann. Phys. 25, 309 (1963); Nuclear Physics 57, 208 (1964).

[8] Burdet, G., M. Giffin, and E. Predazzi: Nuovo cimento 36, 1337 (1965).

[9] Marchenko, V. A.: Doklady Akad. Nauk SSSR 104, 695 (1955). - AgranovicH, Z. S., and V. A. MARChENKo: The inverse problem of scattering theory. New York: Gordon and Breach 1963; translated from the russian edition, Kharkov 1960.

[10] BlažEK, M.: Mat. fyz. čas. SAV 13, 147 (1963).

[11] Acharya, R., and A. BhattacharJee: Nuovo cimento 26, 369 (1962).

[12] BJorken, J. D., and A. Goldberg: Nuovo cimento 16, 539 (1960).

[13] Blažek, M.: Czech. J. Phys. B 12, 497 (1962).

[14] Fowler, M.: Nuovo cimento 20, 478 (1961).

[15] Nyman, E. M.: Nuovo cimento 37, 492 (1965).

[16] Chew, G. F., and S. C. Frautschi: Phys. Rev. 124, 264 (1961).

[17] Moravcsik, M. J., and H. P. Noyes: Ann. Rev. Nucl. Sci. 11, 104 (1961). 